\title{
Research and simulation of the directed double-loop networks with exponential-step
}

\author{
Tao Tao ${ }^{1, ~ a ~}$, Deng-shan $\mathrm{Li}^{1,2, \mathrm{~b} \text { * }}$ \\ ${ }^{1}$ School of computer science and technology, Anhui University of Technology, Maanshan 243000, \\ China \\ ${ }^{2}$ Masteel metrology management devision, Maanshan 243000, China \\ ataotao@ahut.edu.cn, bjopula@163.com
}

\begin{abstract}
Keywords: Directed Double-Loop Networks; Exponential-Step; Random Step; Diameter; Communication Delay
\end{abstract}

\begin{abstract}
In order to reduce the network communication delay in the ultra high speed local area network, based on the topological network structure of "random step" proposed by foreign scholars, to overcome the shortcomings of the "random step", such as complexity of the network topology, disorder of the structure, not easy to connect, not easy to maintain and so on, the network topology structure with exponential-step is proposed. The network topology of the "exponential step" is studied in this paper, and an algorithm for generating exponential-step is proposed in this paper, and using $\mathrm{C}$ to achieve the simulation of the algorithm. Existing data indicate that, the network topology of the "exponential step" not only overcomes the shortcomings of the network structure of the “random step", but also decreases the communication delay.
\end{abstract}

\section{Introduction}

With the continuous progress of science and technology, the application of network is more and more common. As a common topological structure, double loop network is widely used in all kinds of local area networks, the connection of CPUs in supercomputer, and many other places that need super high speed network connection. For the supercomputers, the topology of the network connection between CPUs is very important. Because of the different network topology, the communication delay is different. The communication delay of the network topology will restrict the speed of the cluster computing between the supercomputer's CPUs. Therefore, it is very important to study the topology of the network and improve the communication delay of the network. The communication delay of the double loop network with fixed step is limited, the ring network with random step is proposed by foreigners, but the topological structure of the ring network with random step also has a lot of disadvantages, such as the topology is too complex, difficult to maintain and so on. Based on the topological structure of the ring network with random step, we propose the topological structure of the ring network with exponential step. Preliminary experiments show that, the communication delay of the topological structure of the ring network with the exponential step is much lower than that with the fixed step.

The topological structure of the double loop network is such as that: with such a directed graph $\mathrm{G}(\mathrm{N} ; \mathrm{r}, \mathrm{s})$, mark each vertex as $0,1,2, \ldots, N-1$, two edges are derived from each vertex: $i \rightarrow(i+r)(\bmod N), i \rightarrow(i+s)(\bmod N)$, where $r, s$ is the positive integer which is greater than or equal to 1 , and $r \neq s$. The diameter of the double loop network is marked as $d(N ; r, s)$, the minimum diameter of the double loop network is marked as $d(N)=\min \{d(N ; r, s), 1<r \neq s<N\}$, at present, the minimum value of the minimum diameter is $l_{b}(N)=[\sqrt{3 N}\rceil-2^{[1]}$. A new idea of solving the diameter of double loop network is given in the paper [2-4] -- L shape tile, and how to solve the $\mathrm{L}$ shape tile is given, so that the solution of the diameter of the double loop network becomes more convenient.

Diameter indicates the shortest distance between vertex and vertex of a double loop network, thus the research of diameter becomes a key point of double loop network. Xu Junming found a lot 
of tight optimal network family ${ }^{[5]}$. Paper [6,7] researched and found out a lot of k- tight optimal double loop network infinite family. Paper [8-11] researched the optimal network path and the optimal network families.

\section{Topological structure of the directed double loop network and communication delay of the network}

The topological structure of the directed double loop network is shown in Figure 1. With the method of graph theory, it is marked as $\mathrm{G}(\mathrm{N} ; \mathrm{r}, \mathrm{s}), \mathrm{N}$ is the total number of vertices of a double loop network, and $N \geq 4 . r, s$ is the step of a double loop network, that is the connection between the vertices of the network, and $1 \leq r \neq s<N$. Each vertex sends two lines, that is $[+r]$ line and $[+\mathrm{s}]$ line, called as $[+r]$ edge and $[+s]$ edge.

The directed double loop network is symmetric, distance between any vertex $u$ to $v$, can be replaced by the distance between vertices 0 to $u$ or $v$. Therefore, to study the problem of the diameter of the double loop network, only need to study the distance between vertex 0 to other vertices.

As everyone knows, for ultra high speed local area network, the communication delay is very important. Such as, for the connection between the CPUs of a supercomputer, CPU's computing speed is very fast, but the topology structure of the connection between CPUs is not so good, thus the communication delay between CPU and CPU becomes slow, if so, CPU can not respond timely, the overall performance of the supercomputer will be greatly reduced. Therefore, network with low communication delay is worth studying.

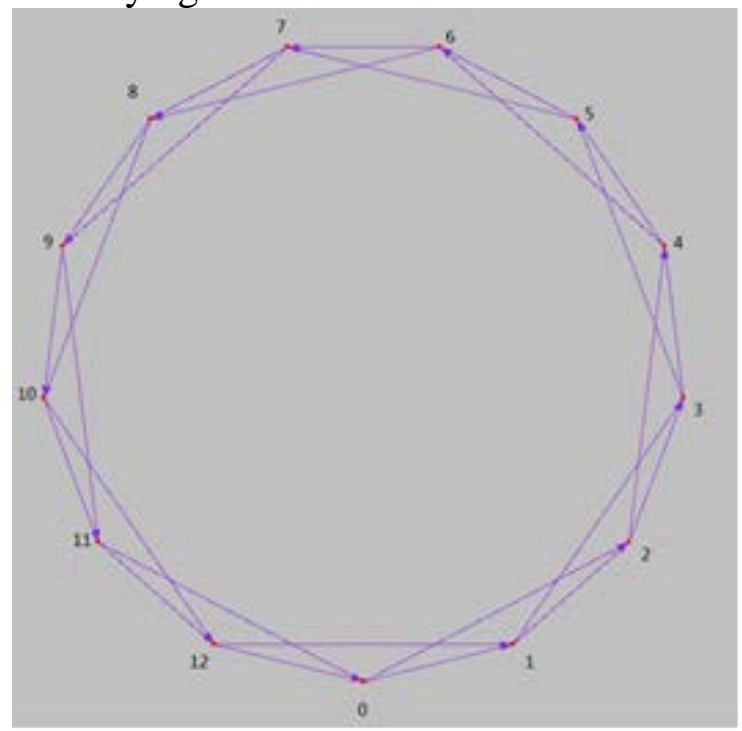

Fig. 1 The topological structure of a directed double loop network G $(13 ; 1,2)$

The $r$ and $s$ is fixed in double loop network with fixed step. The communication delay of the double loop network with fixed step has been unable to break through the delay lower bound given by Wong and Coppersmith ${ }^{[1]}$. Michihiro Koibuchi ${ }^{[12]}$ and others take the random string to connect the ring network, the communication delay is about 300 times lower than that of a fixed step network used on an on-chip system. Michihiro Koibuchi's research gives us a new idea that, to reduce the communication delay of double loop network, we don't need to be confined to a fixed step, and can take the ring network with changing step. Next, we study the double loop network with changing step.

\section{Topological structure of ring network with exponential step}

Double loop network with random step has many advantages, such as the communication delay is lower than those with fixed step, the diameter is small and so on, but ring network with random step also has many disadvantages, such as the connection is confusing, and connection confusion 
leads to difficulties to manage the connection and routing. In view of this, on the basis of random step, we propose a new method-- exponential step.

The generation steps of the topological structure of the directed double loop network with exponential step are as follows:

Step1: first put node 0 , node 1 , node $2, \ldots$, into a circle;

Step2: node 0 sends two directed lines, one points to node 1 , the other points to node 2 . The steps of the two edges are 1 and 2 respectively, that is $r_{0}=1, s_{0}=2$;

Step3: node 1 sends two directed lines, one points to node 3 , the other points to node 4 . The steps of the two edges are 2 and 3 respectively, that is $r_{1}=2, s_{1}=3$;

Step4: and by this analogy, until when $r_{\mathrm{i}} 、 s_{\mathrm{i}}>\left\lceil\frac{N}{2}\right\rceil$, restart drawing from the step value which is 1 and 2, that is $r_{\mathrm{i}}=1, s_{\mathrm{i}}=2$;

Step5: until the in-degree and the out-degree of all nodes are 2, now the drawing is complete. Shown in Figure 2 is the topological structure of a directed double loop network with exponential step of 8 nodes.

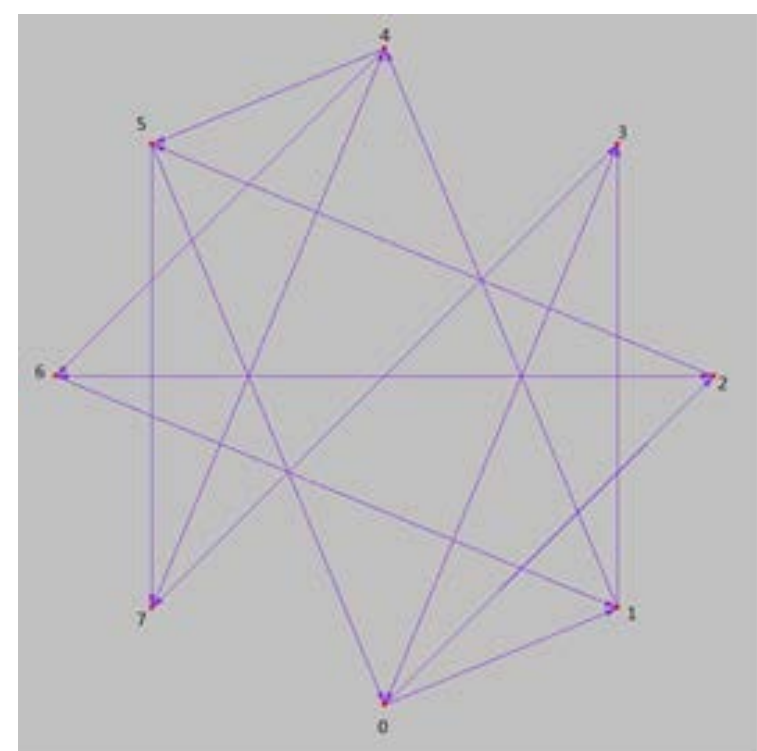

Fig. 2 The topological structure of a directed double loop network with exponential step G(8)

\section{Generation algorithm of ring network with exponential step}

The pseudo code of the exponential step generation algorithm described by $\mathrm{C}$ is as follows: Void GenerateDEG()

\{

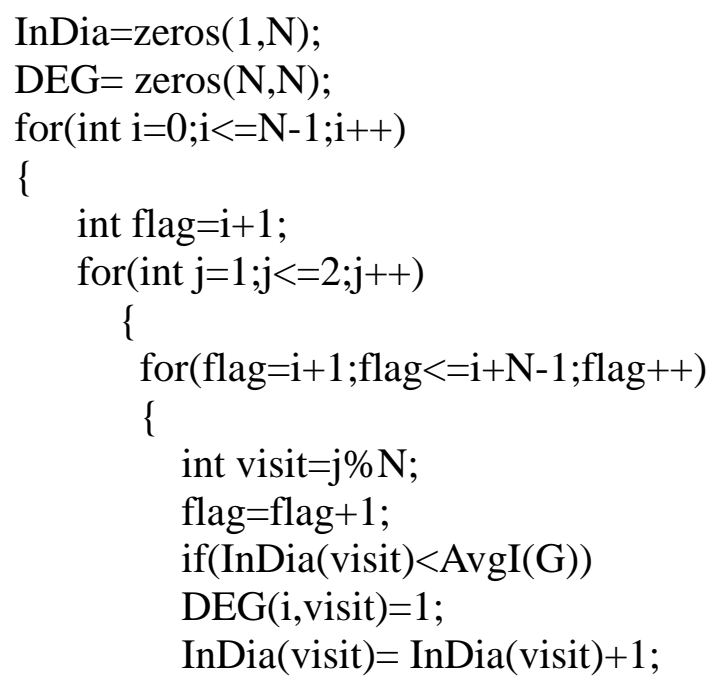




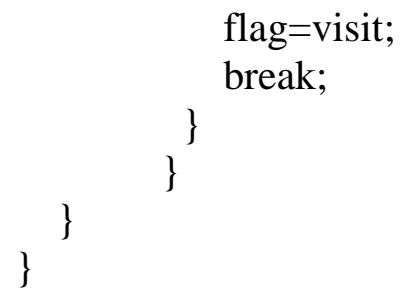

In the above pseudo code, $\operatorname{AvgI}(G)$ is the average in-degree of the drawn topological structure, InDia is a one-dimensional array, used to record the in-degree of every node $I_{0}, I_{1}, I_{2}, \ldots, I_{N-1}$. DEG is a adjacency matrix of the directed double loop network $G(N ; r, s)$, used to record the edge of the exponential step structure of the directed double loop network $\mathrm{G}(\mathrm{N} ; \mathrm{r}, \mathrm{s})$, defined as:

$$
\operatorname{DEG}(i, j)=\left\{\begin{array}{l}
1,<i, j>\in E(G) \\
0,<i, j>\notin E(G)
\end{array}\right.
$$

\section{Simulation result}

In this paper, the topological structure of the directed double loop network with exponential step $\mathrm{G}(\mathrm{N} ; \mathrm{r}, \mathrm{s})$ is simulated by using the $\mathrm{C}$ language, and the generation algorithm of the directed double loop network with exponential step is realized. In this experiment, the value of $N$ is 6,12 respectively, as shown in Figure 3, Figure 4 respectively.

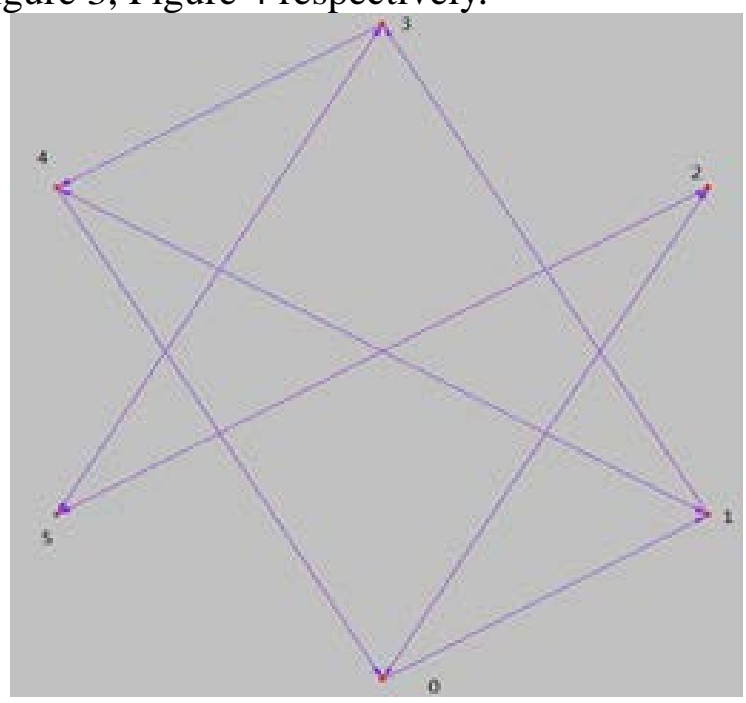

Fig. 3 The topological structure of a directed double loop network with exponential step G(6)

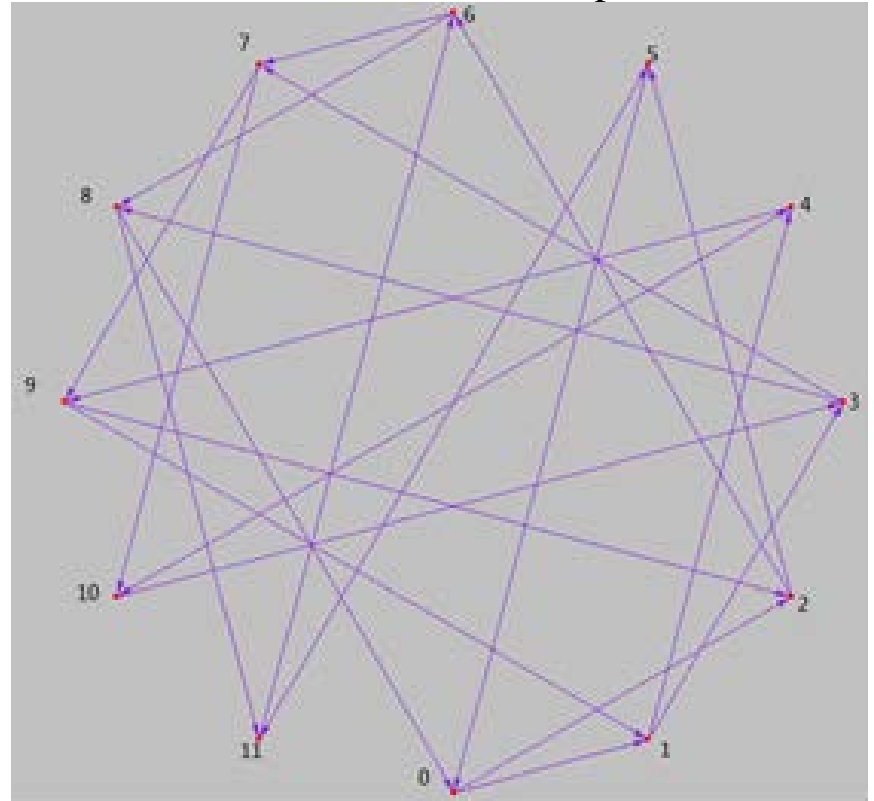

Fig. 4 The topological structure of a directed double loop network with exponential step G(12) 


\section{Conclusions}

This paper starts from the point of reducing the communication delay of the ultra high speed network, uses the theory that communication delay can be reduced by using the random step proposed by Michihiro Koibuchi, proposes the topological structure of double loop network with exponential step, realizes the generation algorithm of the topological structure of exponential step, and simulate the algorithm further.

The specific benefits of the communication delay of the topological structure of double loop network with exponential step, that is, how much communication delay can be reduced by using this topology, and whether the ring network with exponential step has smaller diameter, all these are the future research direction.

\section{Acknowledgments}

The corresponding author is Deng-shan Li, whose work is very important to this paper. This work was financially supported by The National Natural Science Foundation of China under Grant No. 61402009.

\section{Reference}

[1] Wong C K, Coppersmith D. A combinatorial problem related to multimodule organizations [J] Journal of Association Computer, 1974, 21(3): 392-402.

[2] Chen C Y, Hwang F K. The minimum distance diagram of double-loop networks[J]. IEEE Transactions on Computers, 2000,49 (9):977-979.

[3] Chen C Y, James K L, Tang W S. An efficient algorithm to find a double-loop network that realizes a given L-shape[J]. Theoretical Computer Science,2006,359(1/2/3):69-76.

[4] Chen C Y, Hwang F K. Equivalent L-shapes of double-loop networks for the degenerate case[J]. Journal of Interconnection Networks,2000, 1(1):47-60.

[5] Xu Junming, Liu Qi. A class of 4-tight optimal infinite family of double-loop networks[J]. Science In China (Series A), 2003,33(1):71-74.

[6] Zhou Jianqin. 3 infinite families of 6-tight optimal double-loop networks[J]. Journal of University of Science And Technology of China, 2004, 34(4): 431-436.

[7] Zhou Jianqin. On k-tight optimal double-loop networks[J]. Journal of University of Science And Technology of China, 2005,35(6):738-742.

[8] Chan C F, Chen C, Hong Z X. A simple algorithm to find the steps of double-loop networks[J]. Discrete Applied Mathematics, 2002, 121: 61-72.

[9] Liu Huanping, Yang Yixian, Hu Mingceng. On the construction of tight double loop networks[J]. System Engineering Theory and Practice, 2001, 21(12): 72-75.

[10] Chen Zhongxue, Jin Fan, On the [+1]-link-prior shortest path and optimal routing for double-loop networks[J]. Journal of Computer Research and Development, 2001, 38(7):788-792.

[11] Chen Xiebin. An optimal routing algorithm for double loop networks with restricted steps[J]. Chinese Journal of Computers, 2004, 27(5): 596-603.

[12] Koibuchi M, Matsutani H, Amano H, et al. A case for random shortcut topologies for HPC interconnects[C]//Computer Architecture (ISCA), 2012 39th Annual International Symposium on IEEE, 2012: 177-188. 